ABSTRACT: Within 5 weeks, denervated extensor digitorum longus (EDL) muscles of rats lose $66 \%$ of mass, $91 \%$ of force, and $76 \%$ of fiber crosssectional area (CSA). We previously determined the parameters of electrical stimulation for denervated rat EDL muscles to generate tetanic contractions sufficient to maintain mass and force close to control values. Using these parameters, we tested the hypothesis that a range exists for number of contractions per day, below and above which values for mass, maximum force, and fiber CSA are lower than values for innervated control muscles. For 5 weeks, denervated EDL muscles were stimulated to generate between 25 and 5000 contractions daily with contractions separated by constant intervals of rest, repeated $24 \mathrm{~h}$ per day. Force was not maintained with fewer than 200 or more than 800 contractions daily, whereas mass and fiber CSA were not maintained with fewer than 50 contractions daily. Protocols of stimulation that maintain muscle mass and force during prolonged periods of denervation may minimize problems clinically associated with denervation atrophy.

Muscle Nerve 30: 77-86, 2004

\title{
NUMBER OF CONTRACTIONS TO MAINTAIN MASS AND FORCE OF A DENERVATED RAT MUSClE
}

\author{
DOUGLAS E. DOW, PhD, ${ }^{1,2}$ PAUL S. CEDERNA, MD, ${ }^{1,3}$ CHERYL A. HASSETT, ${ }^{1}$ \\ TATIANA Y. KOSTROMINOVA, PhD, ${ }^{1}$ JOHN A. FAULKNER, PhD, ${ }^{1,2}$ \\ and ROBERT G. DENNIS, PhD $1,2,3,4$ \\ ${ }^{1}$ Institute of Gerontology, University of Michigan, Ann Arbor, Michigan, USA \\ ${ }^{2}$ Department of Biomedical Engineering, University of Michigan, Ann Arbor, Michigan, USA \\ ${ }^{3}$ Department of Surgery, University of Michigan, Ann Arbor, Michigan, USA \\ ${ }^{4}$ Department of Mechanical Engineering, University of Michigan, 2350 Hayward, \\ Room 3116, Ann Arbor, Michigan 48109-2125, USA
}

Accepted 12 February 2004

Denervated skeletal muscles show a dramatic loss of maximum force and mass, smaller fiber cross-sectional areas (CSA), ${ }^{14,29}$ larger twitch/tetanus ratios $\left(\mathrm{P}_{\mathrm{t}}: \mathrm{P}_{\mathrm{o}}\right)$, as well as prolonged time-to-peak tensions (TPT) and half-relaxation times (HRT) compared to normal muscles. ${ }^{29,31}$ Electrical stimulation at least partially rectifies these impairments..$^{2,12,31,33}$ The atrophy and weakness that result from prolonged muscle denervation are correlated with a decline in the ability of the muscle to become reinnervated and thus limit the potential for the restoration of motor function following prolonged periods of denerva-

Abbreviations: $C_{d}$, number of tetanic contractions generated each day; CSA, cross-sectional area; CSA $_{m}$, physiological cross-sectional area of the muscle; EDL, extensor digitorum longus muscle; HRT, half-relaxation time; $\mathrm{L}_{\mathrm{f}}$, muscle fiber length; $L_{\circ}$, optimal muscle length; PBS, phosphate-buffered saline; $P_{o}$, maximum isometric tetanic force, maximum force; $P_{t}$, maximum isometric twitch force; $\mathrm{P}_{\mathrm{t}}: \mathrm{P}_{\mathrm{o}}$, twitch-tetanus ratio; TPT, time-to-peak tension Key words: atrophy; contractility; cross-sectional area; EDL; extensor digitorum longus; fiber size; tetanus

Correspondence to: R. G. Dennis; e-mail: bobden@umich.edu

(C) 2004 Wiley Periodicals, Inc.

Published online 2 June 2004 in Wiley InterScience (www.interscience.wiley. com). DOI 10.1002/mus.20054 tion. $5,8,15,22,34$ We reported that an implantable, programmable stimulator and stimulation protocol maintain both muscle mass and maximum force of denervated and stimulated extensor digitorum longus (EDL) muscles of rats at values close to those for innervated, contralateral control muscles. ${ }^{10}$

The specific values of the stimulation variables affect the maintenance of mass and force. ${ }^{1,10,12,16,33}$ For denervated EDL muscles of rats, stimulation protocols that differ in the frequency of pulses, $1,12,16,33$ number of pulses per contraction,,$^{1,10,12,16,33}$ or number of tetanic contractions per day ${ }^{1}$ result in different values for mass and force. In many studies, several stimulation variables were altered simultaneously. Consequently, identification of the effects of a specific stimulation variable, such as the number of contractions per day, is not possible. ${ }^{1,12,16,33} \mathrm{~A}$ balance may occur in the muscle tissue between the positive impact of the generated level of contractile activity and the negative impact of the transfer of electric energy from electrodes into the tissue, as evidenced by damage to muscle tissue following intense protocols of electrical stimulation. ${ }^{26,30}$ Our 
purpose was to determine a range in the number of contractions per day that balanced the positive and negative impacts of either too little or too much electrical stimulation and optimally maintained muscle mass and maximum force. Based on prior work in stimulation for denervated EDL muscles of rats, ${ }^{10}$ we have systematically varied the number of tetanic contractions generated each day $\left(\mathrm{C}_{\mathrm{d}}\right)$, and evaluated the muscle properties after 5 weeks of stimulation. We have tested the hypothesis that a range exists for number of contractions per day, below and above which values for mass, maximum force, and fiber CSA are lower than values for innervated control muscles.

\section{MATERIALS AND METHODS}

Animal Care. The experiment utilized 79 adult male WI/HicksCar rats (Harlan, Indianapolis, Indiana). The animals were housed individually and provided with rat chow and water ad libitum in a restricted access, specific-pathogen-free animal care facility at the University of Michigan. All procedures were conducted in accordance with the guidelines established in the United States Public Health Service Guide for the Care of Laboratory Animals (NIH Publication 85-23) and with the approval of the University Committee on the Use and Care of Animals. For operative procedures, rats were anesthetized with an initial intraperitoneal injection of pentobarbital sodium $(60 \mathrm{mg} / \mathrm{kg}$ ) followed by supplementary doses to maintain a deep state of general anesthesia. All operative procedures were performed using aseptic techniques.

Experimental Groups. Prior studies on denervation or stimulation-denervation of muscle have utilized the unoperated contralateral muscles as controls. ${ }^{5-7,10}$ Utilization of contralateral control muscles has the advantage of minimizing differences in muscle properties due to animal age, body mass, or general state of health but has the disadvantage of not detecting compensatory changes that may have occurred in the contralateral muscles. Consequently, a separate group of control rats was included for this study. Individual animals (rats) were randomly assigned to one of the following four groups: control, sham-wire, denervated, or stimulated-denervated. By including contralateral muscles as an additional control group, the EDL muscles from each animal were divided into five final experimental groups: (1) control, (2) sham-wire, (3) denervated, (4) stimulated-denervated, and (5) contralateral control. For the control group $(n=6$ rats; final age, $5.7 \pm 0.1$ months; weight, $375 \pm 7 \mathrm{~g}$ ), the EDL muscles remained innervated and had no operative procedure prior to evaluation. For the sham-wire group $(n=6$ rats; final age, $6.4 \pm 0.4$ months; weight, $392 \pm 6 \mathrm{~g}$ ) both EDL muscles remained innervated, and electrode wires not connected to a stimulator were implanted 5 weeks prior to final evaluation. For stimulated and sham-wire muscles, the electrode wires looped around the EDL muscles as diagrammed in Figure 1B. For the denervated rats $(n=5$ rats; final age, $6.1 \pm 0.3$ months; weight, $373 \pm 10 \mathrm{~g}$ ) or the stimulated-denervated rats $(n=53$ rats; final age, $6.8 \pm 0.2$ months; weight, $386 \pm 3 \mathrm{~g}$ ), an initial surgery permanently denervated the right EDL muscle. Immediately following, rats in the stimulateddenervated group had a stimulator and electrode wires (Fig. 1A) implanted. ${ }^{10}$ After a 5-week (36.2 \pm 0.3 days) period of recovery following the initial surgery, the denervated or stimulated-denervated muscles were evaluated. For the denervated and stimulated-denervated groups, the left EDL muscle remained innervated, had no operative procedure, and served as a contralateral control muscle.

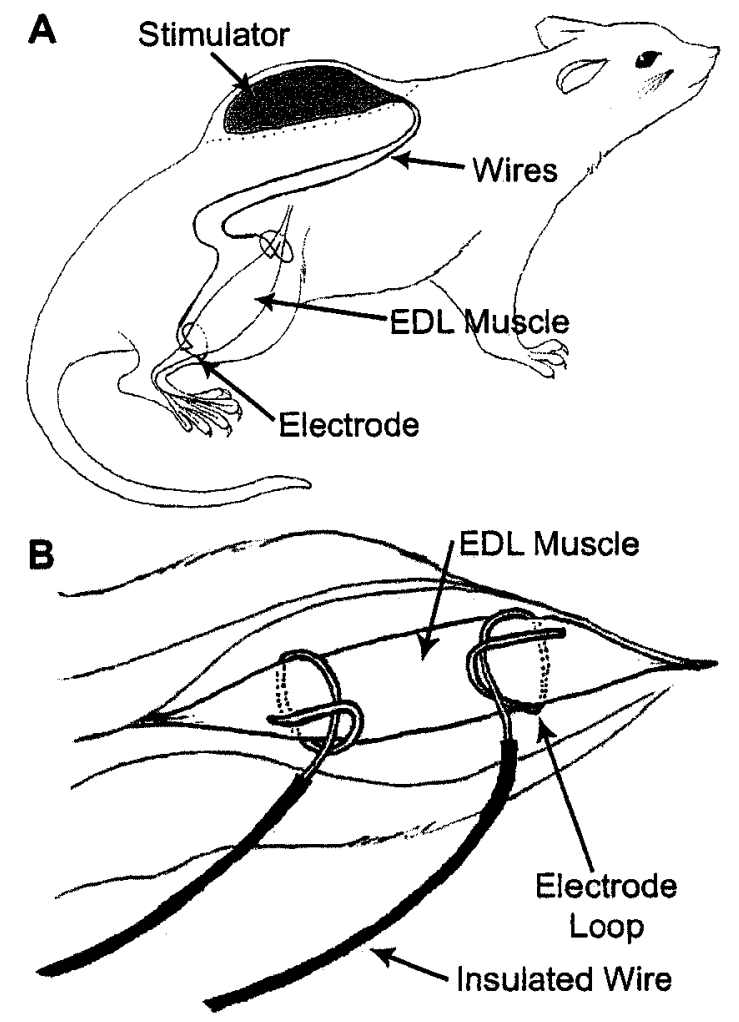

FIGURE 1. Diagram of stimulation system implanted within rat. (A) Placement of implanted stimulator and insulated wires within rat. (B) Electrodes looped around the denervated EDL muscle. Electrodes made by stripping insulation off the ends of the wires. (Illustrations by Megumi Hayashida.) 
Denervation. The EDL muscles in both the denervated and stimulated-denervated groups were denervated according to the procedure described by Carlson and Faulkner.5,7 Briefly, after the rat was anesthetized the sciatic nerve was exposed in the thigh and doubly ligated ( 5 to $10 \mathrm{~mm}$ apart) just distal to the sciatic notch. The intervening segment of sciatic nerve was then resected. The ligated proximal and distal nerve stumps were implanted into separate muscular tissue as far away from each other as possible. This method of denervation has been shown to prevent autoreinnervation of the EDL muscles of rats for at least 22 months. ${ }^{7}$ Sciatic nerve transection has been utilized to denervate the EDL muscle in numerous denervation and stimulationdenervation studies. $2,5,10,12,16,18,25,32$

Stimulator and Electrode Implantation. Stimulators were built, programmed with the desired stimulation protocol, encapsulated, tested and set to sleep mode to prevent stimulator output until activated.9,10 For each rat in the stimulated-denervated group, a stimulator (mass of device, $19 \mathrm{~g}$ ) was implanted into a subcutaneous pocket on the back of the rat (Fig. 1A). The insulated wires (Teflon-coated stainless steel, 36 AWG; AS633, Cooner Wire, Chatsworth, California) passed subcutaneously from the implanted stimulator to the EDL muscle in the anterior compartment of the hindlimb. Electrodes were made by stripping insulation off the end of the wires and looping them around the belly of the EDL muscle, maintaining a distance of at least $10 \mathrm{~mm}$ between the two electrode wires (Fig. 1B). The most proximal electrode was placed on the EDL muscle adjacent to the site of the nerve input that extended distally from the site of the transection in the sciatic nerve described above. In the sham-wire experimental group, for both EDL muscles of each rat the muscles were exposed, electrode wires were stripped of insulation and looped around the muscle, and the insulated ends of the electrode wires extended subcutaneously to the hip region. Only electrode wires were implanted into the sham-wire rats; no stimulator was implanted.

Stimulation Protocol. The stimulator generated a series of pulses and passed current between the pair of electrode wires to depolarize the muscle fibers and generate a tetanic contraction in the denervated EDL muscle. ${ }^{10}$ The values of the fixed stimulation variables used in the current study were based on the natural pattern of activity of innervated EDL muscles of rats $^{19}$ and on protocols obtained from stimulation-denervation studies that have been shown to maintain high levels of mass and force in EDL muscles of rats. ${ }^{10,12,16}$ Each muscle contraction was generated by a train of 20 bipolar pulses at $100 \mathrm{~Hz}$ with an amplitude of $8 \pm 1 \mathrm{~V}$ and 0.4 -ms pulse width. To clarify the pulse definition, the voltage between the two electrode wires during the first $0.2 \mathrm{~ms}$ of the bipolar pulse was $+8 \mathrm{~V}$ and the voltage during the final $0.2 \mathrm{~ms}$ was $-8 \mathrm{~V} .{ }^{10}$ The bipolar pulses were balance and symmetric to prevent net ion flow that would be toxic to the tissue. ${ }^{30}$ These stimulation variables and the physical configuration of the implanted electrodes resulted in a measured current of 6-12 mA between the electrodes during each half of the bipolar pulse.

The stimulated-denervated muscles were subdivided into 10 different groups. Each group received a different protocol of stimulation that consisted of a different number of otherwise identical contractions each day. To determine the effective range of the number of contractions per day to maintain mass and force, the stimulators were programmed to generate $\mathrm{C}_{\mathrm{d}}$ of 25, 50, 100, 200, 300, 500, 800, 1200, 2000 , or 5000 contractions each day. Each stimulator generated the predetermined number of contractions per day $\left(\mathrm{C}_{\mathrm{d}}\right)$, separated by constant intervals of rest. For example, the $\mathrm{C}_{\mathrm{d}}$ of 25 protocol separated each contraction by $57.6 \mathrm{~min}$ of rest, and the $\mathrm{C}_{\mathrm{d}}$ of 5000 protocol separated each contraction by only $17.3 \mathrm{~s}$ of rest. Table 1 lists the period of rest between contractions for each stimulation protocol. This pattern of each contraction followed by a constant interval of rest that was repeated $24 \mathrm{~h}$ per day was also reported in prior stimulation-denervation studies. $2,10,12,16,23$ The stimulation continued for a duration of 5 weeks from 1 day following the denervation and implantation surgery until the final evaluation of the muscle properties.

Monitoring the Rat and Electrically Stimulated Limb. The day after implantation, the health and activity of each rat were verified, and then the stimulator was activated by passing a magnet over the implanted stimulator. ${ }^{10}$ Once activated, the stimulator continuously generated a preprogrammed number of contractions per day. The behavior of the rat was monitored to ensure that the electrical stimulation did not evoke signs of pain during the contractions. The function of the stimulator and the health of the rat were monitored at least once each week while the rat was awake and unrestrained. The function of the stimulator was checked by generating a test muscle contraction. ${ }^{10}$ Lack of any resulting movement in the foot or toes of the stimulated-denervated hindlimb during this test contraction was interpreted as indi- 


\begin{tabular}{|c|c|c|c|c|c|c|c|c|}
\hline$\overline{\mathrm{C}_{\mathrm{d}}{ }^{*}}$ & $\operatorname{Rest}^{\dagger}$ (min) & $n^{\ddagger}$ & Muscle mass (mg) & $P_{\circ}(m N)$ & $\mathrm{sp} P(\mathrm{kPa})$ & $P_{t}: P_{o}$ & TPT (ms) & $\mathrm{HRT}(\mathrm{ms})$ \\
\hline \multicolumn{9}{|c|}{ Control (unoperated animals) } \\
\hline $\begin{array}{l}0 \\
\text { Contr }\end{array}$ & $\begin{array}{c}\text { NA } \\
\text { eral control }\end{array}$ & 12 & $177 \pm 4$ & $2550 \pm 60$ & $196 \pm 10$ & $0.37 \pm 0.01$ & $24.5 \pm 0.6$ & $24.8 \pm 1.0$ \\
\hline $\begin{array}{l}0 \\
\text { Sham }\end{array}$ & NA & 55 & $173 \pm 2$ & $2500 \pm 40$ & $212 \pm 3$ & $0.37 \pm 0.01$ & $25.7 \pm 0.3$ & $26.3 \pm 0.8$ \\
\hline $\begin{array}{l}0 \\
\text { Dener }\end{array}$ & NA & 11 & $197 \pm 8^{\S}$ & $2410 \pm 110$ & $180 \pm 6$ & $0.33 \pm 0.01$ & $23.4 \pm 0.4$ & $25.8 \pm 0.9$ \\
\hline $\begin{array}{l}0 \\
\text { Stimu }\end{array}$ & $\begin{array}{c}\text { NA } \\
\text { d-denervated }\end{array}$ & 5 & $61 \pm 1^{\S}$ & $230 \pm 60^{\S}$ & $55 \pm 15^{\S}$ & $0.72 \pm 0.09^{\S}$ & $49.9 \pm 4.6^{\S}$ & $78.5 \pm 4.8^{\S}$ \\
\hline 25 & 57.6 & 4 & $138 \pm 9^{\S}$ & $1340 \pm 210^{\S}$ & $142 \pm 15^{\S}$ & $0.47 \pm 0.02$ & $27.3 \pm 1.5$ & $35.8 \pm 3.5^{\S}$ \\
\hline 50 & 28.8 & 5 & $162 \pm 8$ & $1810 \pm 90^{\S}$ & $168 \pm 3$ & $0.39 \pm 0.01$ & $24.7 \pm 0.3$ & $30.7 \pm 1.6$ \\
\hline 100 & 14.4 & 6 & $163 \pm 5$ & $2020 \pm 110^{\S}$ & $186 \pm 8$ & $0.37 \pm 0.02$ & $25.2 \pm 0.5$ & $27.6 \pm 1.2$ \\
\hline 200 & 7.2 & 4 & $168 \pm 4$ & $2060 \pm 70$ & $184 \pm 7$ & $0.33 \pm 0.01$ & $24.3 \pm 1.3$ & $25.7 \pm 1.9$ \\
\hline 300 & 4.8 & 9 & $172 \pm 7$ & $2320 \pm 110$ & $199 \pm 5$ & $0.35 \pm 0.01$ & $22.6 \pm 0.4$ & $26.0 \pm 1.4$ \\
\hline 500 & 2.9 & 3 & $179 \pm 20$ & $2050 \pm 60$ & $170 \pm 11$ & $0.32 \pm 0.01$ & $20.1 \pm 1.0$ & $23.5 \pm 2.0$ \\
\hline 800 & 1.8 & 3 & $172 \pm 11$ & $2050 \pm 40$ & $173 \pm 6$ & $0.30 \pm 0.01$ & $23.7 \pm 0.5$ & $27.8 \pm 2.8$ \\
\hline 1200 & 1.2 & 3 & $184 \pm 15$ & $1910 \pm 60$ & $161 \pm 17$ & $0.33 \pm 0.03$ & $24.4 \pm 1.2$ & $27.9 \pm 1.7$ \\
\hline 2000 & 0.72 & 3 & $166 \pm 10$ & $1830 \pm 160^{\S}$ & $161 \pm 11$ & $0.28 \pm 0.01$ & $26.2 \pm 0.6$ & $29.1 \pm 1.2$ \\
\hline 5000 & 0.29 & 3 & $153 \pm 6$ & $1680 \pm 80^{\S}$ & $165 \pm 12$ & $0.27 \pm 0.00$ & $23.5 \pm 1.7$ & $31.0 \pm 3.6$ \\
\hline
\end{tabular}

*Number of tetanic contractions generated each day.

${ }^{+}$Number of minutes separating each contraction, repeated $24 \mathrm{~h}$ per day.

${ }^{\ddagger}$ Number of EDL muscles evaluated for that group.

${ }^{\S}$ Difference from values of control muscles.

Values reported as mean $\pm S E$.

cating a defective stimulator. Data recorded from stimulated-denervated muscles having a defective stimulator were excluded from further analysis.

Electrophysiological Evaluation. The contractile properties of the EDL muscles were evaluated in vitro according to the procedure of Faulkner et al., 5,7,10,13 described briefly here. After each rat was anesthetized, each EDL muscle was exposed, sutures were placed around the distal and proximal tendons, and the tendons were severed. The muscle was removed from the rat and placed in an oxygenated tissue bath with a Krebs-Ringer bicarbonate solution. In the bath, one tendon of the muscle was secured to a fixed post and the other to a force transducer (model BG-1000; Kulite Semiconductor Products Inc., Leonia, New Jersey). Platinum electrode plates were immersed in the bath on either side of the muscle, and the muscle was electrically stimulated by square-wave pulses having a duration of $0.2 \mathrm{~ms}$. The voltage and muscle length were adjusted to determine the maximum isometric twitch force $\left(P_{t}\right)$. At these settings for voltage and muscle length that generated $\mathrm{P}_{t}$, values for this optimal muscle length $\left(\mathrm{L}_{\mathrm{o}}\right)$, as well as $\mathrm{P}_{\mathrm{t}}$, TPT, and HRT were recorded. Muscle fiber length $\left(\mathrm{L}_{\mathrm{f}}\right)$ was calculated as being equal to $0.40 * \mathrm{~L}_{\mathrm{o}}$, using the value of 0.40 for the $\mathrm{L}_{\mathrm{f}} / \mathrm{L}_{\mathrm{o}}$ ratio of EDL muscles of WI/HicksCar rats. ${ }^{6}$ Maximum force $\left(\mathrm{P}_{\mathrm{o}}\right)$ was determined for te- tanic, isometric contractions as previously reported.5,7,10,13 The twitch/tetanus ratio, $\mathrm{P}_{\mathrm{t}}: \mathrm{P}_{\mathrm{o}}$, was calculated by dividing the value of $\mathrm{P}_{t}$ by the value of $\mathrm{P}_{\mathrm{o}}$. Specific force was calculated as equal to $\mathrm{P}_{\mathrm{o}} /$ $\mathrm{CSA}_{\mathrm{m}}$, where $\mathrm{CSA}_{\mathrm{m}}$ is the physiological CSA of the muscle. The $\mathrm{CSA}_{\mathrm{m}}$ was calculated as $\mathrm{m} * \cos \theta /(\rho *$ $\mathrm{L}_{\mathrm{f}}$ ), where $\mathrm{m}$ is the muscle mass in $\mathrm{mg}, \theta$ is the pennation angle (approximated as $180^{\circ}$ ), and $\rho$ is the density of mammalian skeletal muscle $(1.06 \mathrm{mg} /$ $\mathrm{mm}^{3}$ ). After both EDL muscles had been removed, the rats were administered an overdose of anesthesia and the thoracic cavity was opened to ensure that the rats were euthanized.

Preparation of Muscle Tissue. After measurement of contractile properties, the muscle was removed from the bath, tendons were trimmed, and the muscle was blotted and weighed. Muscles were placed into Tissue Freezing Medium (Triangle Biomedical Sciences, Durham, North Carolina), quickly frozen by immersion in isopentane cooled by dry ice, and then stored at $-70^{\circ} \mathrm{C}$.

Postexplant Evaluation of Stimulator. At the time of euthanasia, the electrical stimulators were removed, washed, and evaluated for proper functioning. A digital oscilloscope was used to verify stimulator output. A stimulator was considered defective if the waveform exhibited any of the following conditions: 
monopolar instead of bipolar pulses, pulse width longer than $0.6 \mathrm{~ms}$, or amplitude less than $5 \mathrm{~V}$. In the event that a defective stimulator unit was identified, the corresponding data and muscle tissues were discarded without further analysis.

Laminin Labeling of Sections. To clearly visualize individual muscle fibers, laminin staining was employed. Transverse frozen sections $(9.0-\mu \mathrm{m}$ thickness) were obtained from the middle portion of the EDL muscle with a cryostat, mounted on glass slides, and placed in a freezer at $-70^{\circ} \mathrm{C}$ for storage. Before immunohistochemical staining, sections were washed in double-distilled water and fixed in cooled $100 \%$ methanol. Slides were allowed to air dry and were then rehydrated in phosphate-buffered saline (PBS). Labeling with antilaminin (mouse monoclonal antirat laminin B2 clone D18; DSHB, University of Iowa, Iowa City) primary antibody was carried out at room temperature for $3 \mathrm{~h}$. After incubation, sections were washed in PBS and stained with secondary antibody, FITC-conjugated goat antimouse, at room temperature for $45 \mathrm{~min}$. Sections were then rinsed in PBS and mounted in Vectashield mounting medium (Vector Laboratories, Burlingame, California). Observation and photography of the sections were made with a Zeiss Axiophot-2 Universal Microscope (Carl Zeiss Inc., Hallbergmoos, Germany).

Evaluation of Fiber CSA. Transverse frozen sections (10-50 $\mathrm{mm}$ in thickness) of each muscle were cut with a cryostat through the middle portion of the muscle. The sections were stained with hematoxylin and eosin and examined by light microscopy (Leitz Laborlux; Leica, Wetzlar, Germany). A video camera (Diagnostic Instruments, Sterling Heights, Michigan) and image analysis software (Bioquant Imaging System, RM Biometrics, Nashville, Tennessee) running on a personal computer were utilized to systematically capture images at $25 \times$ magnifications in a row-by-row fashion that would constitute a photomontage of the entire cross section of the muscle. A $4 \times 4$ grid was superimposed on each image. All cells whose area was at least $50 \%$ within the innermost four boxes of the grid were selected and the CSA of each fiber in the sample was manually determined using the Bioquant software. For each muscle in which fiber CSAs were to be determined, the CSA of at least 500 fibers were determined and were included in the analysis.

Statistics. Statistical analysis was performed using SPSS software (SPSS Inc., Chicago, Illinois), including calculations of mean and standard error (SE).
For the dependent variables of muscle mass, maximum force, specific force, TPT, HRT, $\mathrm{L}_{\mathrm{f}}$, and $\mathrm{P}_{\mathrm{t}}: \mathrm{P}_{\mathrm{o}}$, a one-way analysis of variance (ANOVA) was used to compare differences between the different experimental groups. When a significant main effect was found, the Tukey test was used for post hoc analysis and the 0.05 level of probability was used to signify statistical significance. For analysis of the fiber CSAs, as the values did not have a normal distribution, nonparametric analysis was performed using the Kruksal-Wallis test to determine whether differences existed between the groups (0.05 significance level). If differences existed, each of the possible 15 pairs of six groups was tested for difference using the MannWhitney test. A Bonferroni correction was used to adjust the criterion level, such that $0.05 / 15$ equals 0.0033 was used as the significance level for the Mann-Whitney $U$ tests.

\section{RESULTS}

Inclusion of Data. Data were recorded for EDL muscles from each of the five experimental groups of EDL muscles: control (innervated muscles from unoperated rats), sham-wire (innervated with passive electrodes), denervated, stimulated-denervated, and contralateral control (unoperated leg contralateral of denervated or stimulated-denervated muscles). Of the 79 rats that were involved in the experiment, 8 rats did not survive the initial surgery, 1 rat was euthanized after the stimulator became defective, and 70 rats completed the study having their right and left EDL muscles evaluated. Of the 70 left EDL muscles, data for 1 sham-wire and 3 contralateral control EDL muscles were discarded because the evaluation procedure was not correctly completed. Of the 70 right EDL muscles, data from 10 of the 53 stimulated-denervated EDL muscles were discarded. Of the 10 muscles discarded, 8 had a defective stimulator, 1 rat had an ulcer on the heel of the denervated hindlimb, and 1 muscle had an unsuccessful evaluation procedure. Including the defective stimulator of the rat that was euthanized prior to muscle evaluation, $17 \%$ (9 of 54) of the stimulators used in the study became defective. For each experimental group, only data from muscles that were not discarded, as discussed above, were analyzed (Tables 1 and 2; Figs. 2 and 3).

Whole-Muscle Properties. Values for control muscles (unoperated animals) and contralateral control muscles were not different from one another for muscle mass, maximum force, specific force, $\mathrm{P}_{\mathrm{t}}: \mathrm{P}_{\mathrm{o}}$, TPT, or HRT (Table 1). Any compensatory changes 


\begin{tabular}{|c|c|c|c|}
\hline $\mathrm{C}_{\mathrm{d}}{ }^{*}$ & $\begin{array}{c}\text { No. of } \\
\text { muscles }\end{array}$ & $\begin{array}{l}\text { No. of fibers per } \\
\text { muscle }\end{array}$ & Fiber CSA $\left(\mu \mathrm{m}^{2}\right)$ \\
\hline \multicolumn{4}{|c|}{ Contralateral control } \\
\hline $\begin{array}{l}0 \\
\text { Dener }\end{array}$ & 7 & $700 \pm 31$ & $2790 \pm 90$ \\
\hline 0 & 5 & $901 \pm 85$ & $670 \pm 60^{\dagger}$ \\
\hline \multicolumn{4}{|c|}{ Stimulated-denervated } \\
\hline 25 & 4 & $1021 \pm 74$ & $1680 \pm 180^{\dagger}$ \\
\hline 50 & 4 & $813 \pm 83$ & $2510 \pm 190^{\dagger}$ \\
\hline 300 & 4 & $787 \pm 85$ & $3270 \pm 310^{\dagger}$ \\
\hline 5000 & 3 & $695 \pm 47$ & $2750 \pm 220$ \\
\hline
\end{tabular}

*Number of contractions per day generated by the electrical stimulation. ${ }^{+}$Difference with values for contralateral control muscles.

Values are given with SE. The values for each group listed in the table were different from the values for all other groups, except that contralateral control and stimulated-denervated with $C_{d}$ of 5000 had values that were not different from one another.

in these properties that may have occurred in the innervated muscles contralateral to the denervated or stimulated-denervated muscles were not large enough to allow detection in the current study. Compared with control muscles, the presence of the passive electrode wires on innervated muscles of the sham-wire group did not affect the maximum force, specific force, $\mathrm{P}_{\mathrm{t}}: \mathrm{P}_{\mathrm{o}}$, TPT, HRT or fiber length. The larger mass of the sham-wire muscles (Table 1) may be due to additional connective tissue that encapsulated the electrode wires.

At 5 weeks, the denervated muscles $\left(C_{d}, 0\right)$ showed dramatic declines in muscle mass to $34 \%$ of control values and maximum force to $9 \%$ of control values (Fig. 2; Table 1). Compared with the denervated muscles $\left(C_{d}, 0\right)$, each of the experimental groups of stimulated-denervated muscles $\left(C_{d}>0\right.$; Fig. 2) maintained higher values of both mass and force. When $\mathrm{C}_{d}$ was set in the range of 200 to 800 contractions per day, mass and force were maintained at values not different from values for control muscles (Fig. 2; Table 1). Therefore, the stimulation protocols with $\mathrm{C}_{\mathrm{d}}$ of 200 to 800 , that maintained mass and force, were designated as optimal protocols for denervated EDL muscles of rats. In contrast, the stimulation protocols with $\mathrm{C}_{\mathrm{d}}<200$ or $\mathrm{C}_{\mathrm{d}}>800$ were designated as suboptimal because the maximum force was lower than control values (Fig. 2; Table 1). Mass was maintained at values not different from control values for all stimulation-denervation groups having $\mathrm{C}_{\mathrm{d}}>25$. A suboptimal protocol, with $\mathrm{C}_{\mathrm{d}}$ of 25 , resulted in both muscle mass $(80 \%$ of control) and maximum force (54\% of control) at lower levels than the control muscles, though still considerably higher than those of denervated muscles (Fig. 2; Table 1).
None of the experimental interventions had any effect on muscle length $\left(\mathrm{L}_{\mathrm{o}}\right)$ or the calculated fiber length $\left(\mathrm{L}_{\mathrm{f}}\right)$. The twitch/tetanus ratio, $\mathrm{P}_{\mathrm{t}}: \mathrm{P}_{\mathrm{o}}$, of the denervated muscles were higher than those for control muscles, but each group of stimulated-denervated muscles maintained $\mathrm{P}_{t}: \mathrm{P}_{\mathrm{o}}$ not different from control values (Table 1). The durations of TPT and HRT of the denervated muscles were about twofold or threefold longer than the durations of control muscles (Table 1). All the groups of stimulateddenervated muscles had values of TPT and HRT not different from control values, except that HRT was higher for the muscles receiving the suboptimal protocol having the least number of contractions $\left(\mathrm{C}_{\mathrm{d}}\right.$, 25; Table 1). No group of stimulated-denervated muscles had values of TPT or HRT that differed from values for any other stimulated-denervated group (Table 1).

Morphology, Laminin-Labeled Sections, and Muscle Fiber CSA. Fibers of denervated muscles (Figs. 3B and $3 \mathrm{~F}$ ) had smaller CSAs than did fibers of contralateral control muscles (Figs. 3A and 3E). The stimulated-denervated muscles (Figs. 3C, 3D, 3G,

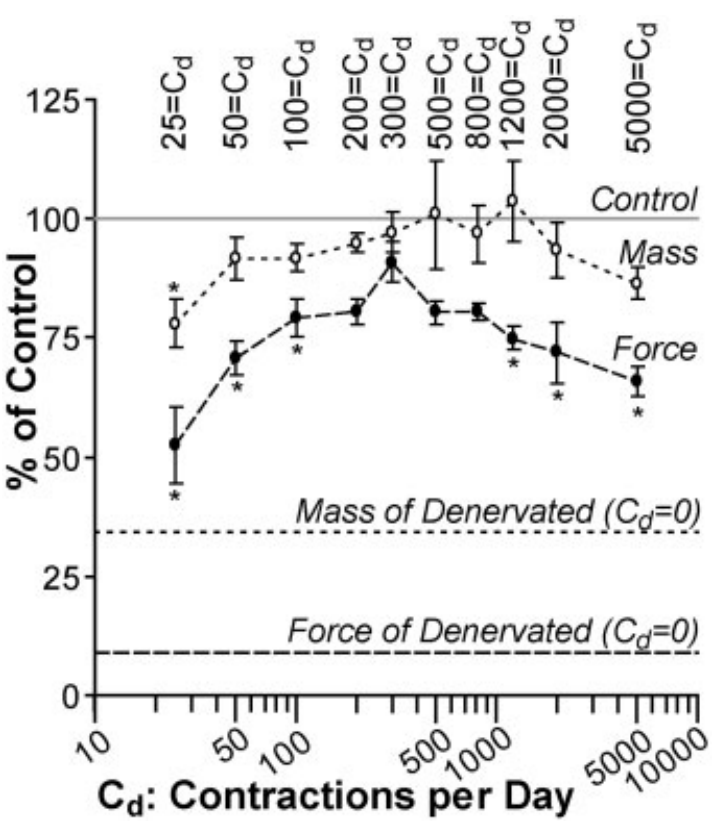

FIGURE 2. Muscle mass and maximum force are plotted as percent of control values (unoperated rats) for the EDL muscles that had been stimulated-denervated for 5 weeks. The top horizontal line, at $100 \%$, indicates the level of mass and force of control muscles (unoperated rats). The lower two, dashed, horizontal lines indicate the level of mass (34\%) and force $(9 \%)$ maintained by denervated muscles that received no electrical stimulation. Error bars are standard error (SE) measure. The asterisk indicates difference with values for control muscles $(P<$ 0.05). 


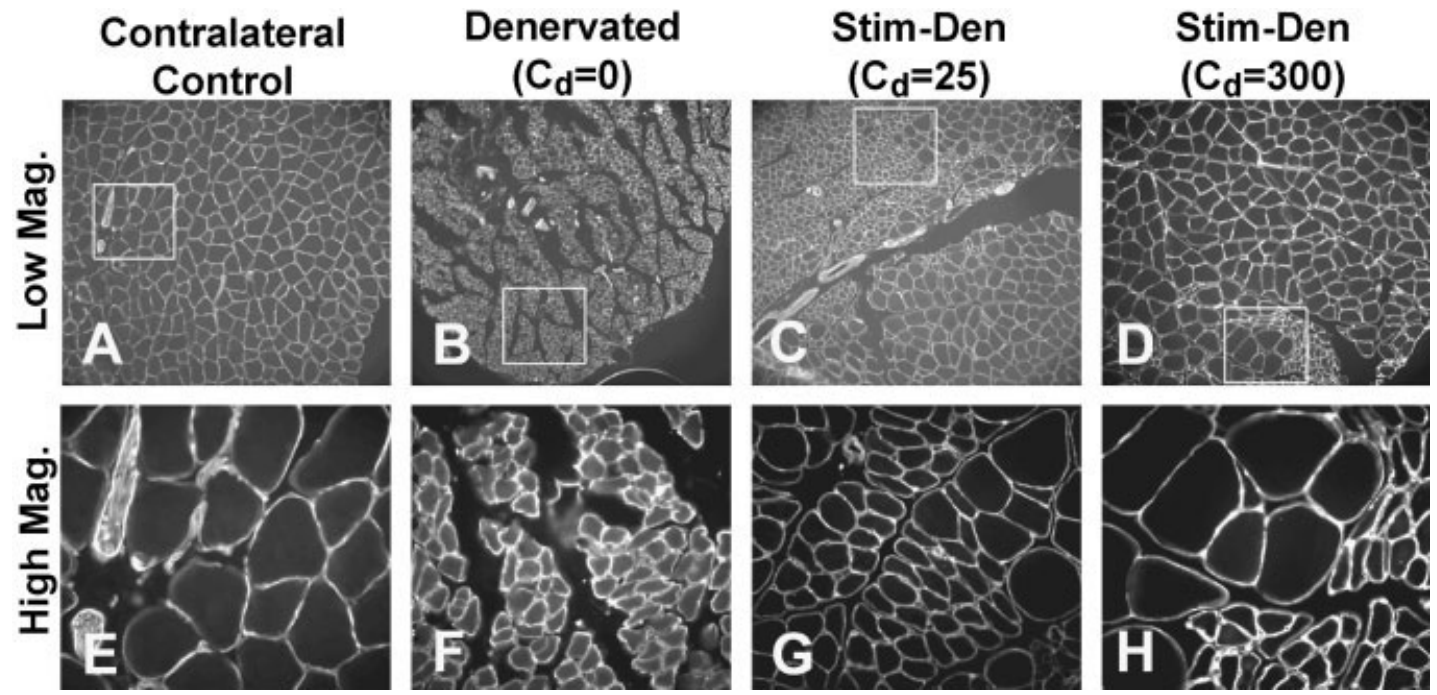

FIGURE 3. A two- $X$-four arrangement of immunofluorescent photomicrographs showing the expression of laminin in contralateral control $(A, E)$, denervated $(B, F)$, and stimulated-denervated (C, D, G, H) EDL muscles of rats. The laminin-stained fibers in the top row (A-D) are at a lower original magnification $(\times 20)$ than those in the second row $(\times 63)(E-H)$. For each column, a subregion visible in top row $(A-D)$ is displayed in the second row $(E-H)$ at a higher magnification. Representative regions for each muscle type are shown. The third column $(\mathbf{C}, \mathbf{G})$ shows a stimulated-denervated muscle $\left(\mathrm{C}_{\mathrm{d}}, 25\right)$ that was designated as having received a suboptimal protocol of stimulation because mass and force were maintained at lower values than values for control muscles. The fourth column (D, H) shows a stimulated-denervated muscle $\left(C_{d}, 300\right)$ that was designated as having received an optimal protocol. Note that images of the stimulated-denervated muscles (C, D) have subregions of smaller fibers that appear similar to those in denervated muscles (B, F) and subregions of larger fibers that appear similar to those in contralateral control muscles (A, E).

and $3 \mathrm{H})$ contained some fibers with small CSAs that were apparently similar to fibers of denervated muscles (Figs. 3B and 3F) and contained fibers with large CSAs that were apparently similar to fibers of control muscles (Figs. 3A and 3E). The suboptimally stimulated muscles $\left(\mathrm{C}_{\mathrm{d}}, 25\right.$; Figs. $3 \mathrm{C}$ and $\left.3 \mathrm{G}\right)$ contained larger regions that consisted mainly of smaller fibers than the optimally stimulated muscles $\left(\mathrm{C}_{\mathrm{d}}, 300\right.$; Figs. $3 \mathrm{D}$ and $3 \mathrm{H})$. Following 5 weeks of denervation, the mean fiber CSA decreased to 24\%. Each of our protocols of electrical stimulation attenuated this decrease (Table 2). For stimulation protocols up through $\mathrm{C}_{\mathrm{d}}$ of 300 , an increase in the number of contractions resulted in an increase in mean fiber CSA. Fiber hypertrophy was observed at a $\mathrm{C}_{\mathrm{d}}$ of 300 , compared with fibers of contralateral control muscles. In contrast, mean fiber hypertrophy was not observed at the high end of the range of generated contractions $\left(\mathrm{C}_{\mathrm{d}}, 5000\right.$; Table 2$)$.

The frequency distribution of CSAs of fibers from contralateral control muscles fell into a Gaussian distribution (Fig. 4), with only a tiny fraction of fibers (1-2\%) having CSAs in either the smallest $\left(<1000 \mu \mathrm{m}^{2}\right)$ or largest $\left(>5000 \mu \mathrm{m}^{2}\right)$ range. In contrast, denervated muscles had a large majority $(89 \%)$ of fibers within the smallest range $(<1000$ $\mu \mathrm{m}^{2}$ ) with almost no fibers within the largest four ranges $\left(>2000 \mu \mathrm{m}^{2}\right)$. All stimulated-denervated muscles had a population of fibers in the smallest range and some in the larger ranges of CSAs. The distributions of fibers of stimulated-denervated muscles having $\mathrm{C}_{\mathrm{d}}$ of 25,50 , or 300 were different from the distributions of either contralateral control or denervated muscles. Muscles stimulated by a suboptimal protocol $\left(\mathrm{C}_{\mathrm{d}}, 25\right)$ had many more fibers in the smallest range (50\%) compared with muscles stimulated by an optimal protocol $\left(\mathrm{C}_{\mathrm{d}}, 300 ; 16 \%\right)$. Muscles from an optimal group $\left(\mathrm{C}_{\mathrm{d}}, 300\right)$ had a population of fibers in the largest range, indicating some fiber hypertrophy compared with the control muscles.

\section{DISCUSSION}

Our hypothesis was supported by the observations that: (1) values for muscle mass, maximum force, and fiber CSA were not lower than control values (unoperated rats) for stimulated-denervated muscles having $\mathrm{C}_{\mathrm{d}}$ of 200 to 800 contractions per day; (2) for $\mathrm{C}_{\mathrm{d}}<200$, mass, force, and fiber CSA were lower than control values (specifically true for all three measures at $\mathrm{C}_{\mathrm{d}}$ of 25); and (3) for $\mathrm{C}_{\mathrm{d}}>800$, force was lower than control values. Our hypothesis was not supported by the observations that mass and fiber CSA were not lower than control values for $\mathrm{C}_{\mathrm{d}}>800$ up through $\mathrm{C}_{\mathrm{d}}$ of 5000 . Our understanding 


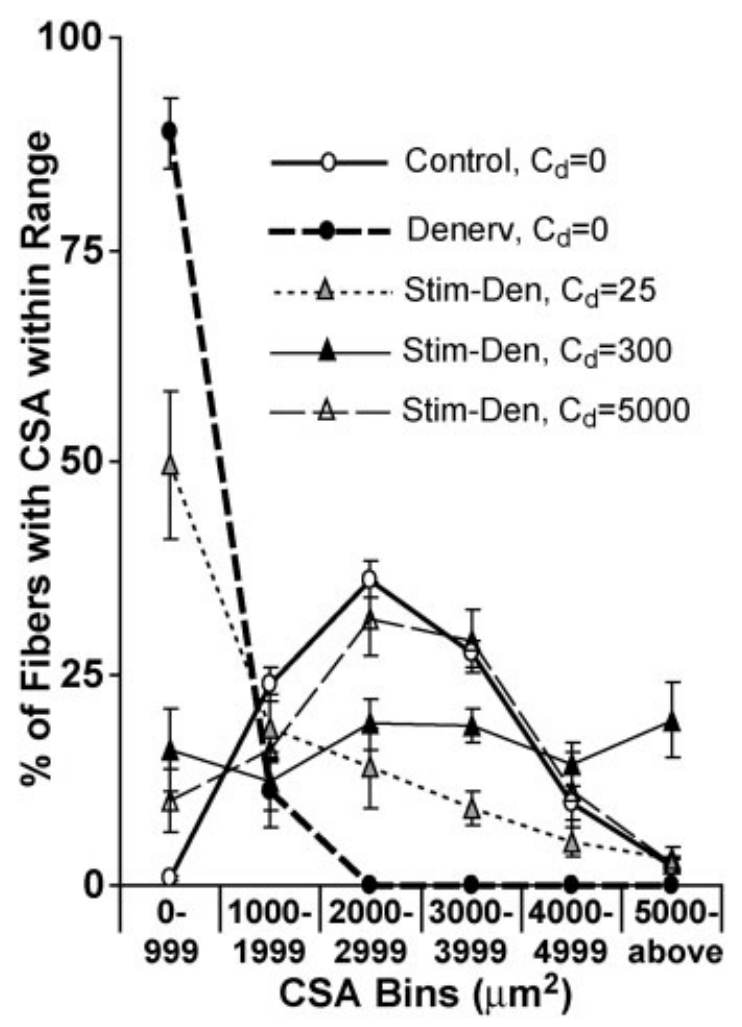

FIGURE 4. Frequency of distribution of muscle fibers having CSAs within the specified ranges for control, denervated, and stimulated-denervated at $C_{d}$ of 25,300 , and 5000. For each experimental group, the average percent of fibers having CSAs within each listed range of CSAs was the average percent for several muscles (Table 2). Error bars are standard error (SE) measure.

is that stimulated-denervated muscles that had $\mathrm{C}_{\mathrm{d}}$ of $<200$ did not have sufficient contractile activity to prevent denervation atrophy. Except for the lower values of force, $C_{d}>800$ up to $C_{d}$ of 5000 contractions per day did not generate excessive levels of contractile activity or energy transfer from the electrodes to inhibit the maintenance of mass and fiber CSA. The effect of even higher numbers of contractions $\left(C_{d}>5000\right)$ was not determined in this study.

The observation that $\mathrm{C}_{\mathrm{d}}$ in the range of 200 to 800 contractions per day maintained muscle mass and maximum force at values not different from control values improves upon our previous report that tested only 100 and 300 contractions per day. ${ }^{10}$ The numbers of contractions per day maintained both mass and force at levels higher than those given in previous reports of electrically stimulated-denervation EDL muscles of rats. ${ }^{2,12,16,18,33}$ In agreement with Eken and Gundersen, ${ }^{12}$ the optimal protocol also maintained $\mathrm{P}_{\mathrm{t}}: \mathrm{P}_{\mathrm{o}}$, TPT, and HRT at values not different from control levels, suggesting that many cellular mechanisms within muscle fibers are main- tained by electrically induced activity, even in the absence of nerve-derived trophic factors.

Insufficient contractile activity has been cited as a primary regulatory signal involved in the loss of mass, force, and fiber size in denervated muscles having less than the optimal range of the number of generated tetanic contractions. ${ }^{4,12,16,17,33}$ The concept is supported by a wide range of protocols for electrical stimulation that generate tetanic contractions in denervated muscles outside the optimal range that still retards the loss of mass, ${ }^{17}$ force ${ }^{2,8,12,16,18,33,34}$ and fiber CSAs. ${ }^{27,31}$ Buffelli et al. ${ }^{4}$ reported that innervated but noncontracting (by means of a nerve conduction block) muscles undergo a decline in mass, force, and fiber size indistinguishable from the declines in denervated muscles. Our study has clarified that a relation exists in denervated muscles between the number of generated tetanic contractions and the resulting mass, force, and fiber size up to levels not lower than values of control muscles. For the lower end of the range of $\mathrm{C}_{\mathrm{d}}$, our observations support a direct relation between the number of tetanic contractions and the maintained level of mass $\left(C_{d}\right.$ of $\left.0,25,50\right)$, force $\left(\mathrm{C}_{\mathrm{d}}\right.$ of $\left.0,25,50,100,200\right)$, and fiber size $\left(\mathrm{C}_{\mathrm{d}}\right.$ of 0,25 , $50,300)$ for stimulated-denervated EDL muscles of rats.

The mechanism by which too many electrically generated contractions per day in stimulateddenervated muscles failed to maintain maximum force as high as protocols within the optimal range is not clear. Greater contractile activity increases metabolic demand and induces smaller fibers and a reduction in muscle force. ${ }^{12,33}$ Greater numbers of contractions per day may also increase tissue damage as a result of increased net ion flux caused by imperfectly balanced current flow during both halves of each bipolar pulse. ${ }^{30}$ Stimulation protocols that more closely resemble the normal contractile activity of a muscle are more successful in maintaining mass and force. ${ }^{12,16,33}$ The optimum range for $\mathrm{C}_{\mathrm{d}}$ of 200 to 800 contractions per day falls near the lower end of the typical range for contractile activity in EDL muscles of rats. ${ }^{19}$ The observations may reflect a balance between adequate activity level and negative factors associated with electrical stimulation. Nevertheless, $\mathrm{C}_{\mathrm{d}}$ of 1000 to 5000 did maintain maximum force well above values for denervated muscles and maintained mass, fiber CSA, specific force, $\mathrm{P}_{\mathrm{t}}: \mathrm{P}_{\mathrm{o}}$, TPT, and HRT at values not lower than those for control muscles.

The lower end of the optimal range has advantages for battery-powered applications. All other parameters being held constant, the total energy cost per day for the stimulator is approximately propor- 
tional to the number of generated pulses. ${ }^{9,10}$ Fewer contractions generated per day translates to reduced energy consumption, longer battery life, and lower level of energy infused into the tissue that may result in tissue damage. ${ }^{26,30}$ For the other stimulation variables fixed as in this study, 200 contractions per day would be a good design choice to maintain mass, force, and fiber CSA while minimizing energy transfer that may negatively affect the tissue and decrease battery life.

For denervated muscles stimulated with an optimal protocol, the mean muscle fiber CSA was higher than CSAs in control muscles and a subpopulation of fibers $(\sim 20 \%)$ had CSAs larger than those typically found in control muscles. The observations provide evidence for stimulation-induced hypertrophy of some of the muscle fibers, even in the absence of innervation. Nevertheless, the optimally stimulateddenervated muscles still contained a subpopulation $(\sim 16 \%)$ of smaller fibers having CSAs similar to fibers that predominate in denervated muscles and are rare in control muscles. The subpopulation of fibers may not have experienced sufficient stimulation to depolarize the fibers, generate tetanic contractions, and maintain fiber CSAs. The nonuniform distribution of fiber CSAs may be due to either a nonuniform voltage field across the muscle fibers or a higher threshold for individual muscle fiber excitability. ${ }^{11}$ Further optimization of the electrode geometry and placement and stimulation pulse width and amplitude may be necessary to reduce the number of insufficiently stimulated fibers. ${ }^{10,11}$

Denervation and stimulation-denervation studies have used different types of muscles as the control group, including muscles from unoperated animals, ${ }^{8}$ muscles in an unoperated leg contralateral to the experimental muscle, ${ }^{5-7,10,12,16,29}$ or variations of sham-operated or sham-wire muscles. Utilization of contralateral control muscles has the advantage of minimizing differences in muscle properties due to animal age, body mass, or general state of health but also has the disadvantage of not detecting compensatory changes that may have occurred in the contralateral muscles. For the EDL muscles of rats used in stimulation-denervation studies similar to the current one, the observations of no difference between values of muscle mass, maximum force, specific force, $\mathrm{P}_{\mathrm{t}}: \mathrm{P}_{\mathrm{o}}$, TPT, or HRT support the utilization of either contralateral muscles or muscles of unoperated animals as a control for the stimulateddenervated EDL muscles. A reason that EDL muscles contralateral to denervated muscles did not show an increase in mass may be that the EDL muscle is not a weight-bearing muscle. The sham-wire muscles did have a higher mass than either the contralateral control or control (unoperated animals) muscles in the current study. A reason for this may be the presence of connective tissue around the electrodes adjacent to the muscle. It is possible that all of the stimulated-denervated muscles have such an increase in mass because of the presence of the electrodes. Because contractile force would not be enhanced by the presence of additional connective tissue on the muscle, measures of force may be a better indicator of the state of the stimulateddenervated muscles than muscle mass.

Protocols of stimulation that minimize the atrophy and weakness of denervated muscles may be effective as a treatment to counter other negative effects of denervation. ${ }^{21,24,34}$ Prolonged periods of denervation prior to reinnervation decrease the ability of muscles to recover mass and maximum force, ${ }^{5,15,22}$ but electrical stimulation during the period of denervation may enhance the recovery of motor function following reinnervation. $8,17,28,34$ Another application is to prevent secondary complications related to disuse atrophy of paralyzed or denervated muscles. Flaccid hemiparalysis following stroke may lead to painful shoulder subluxation, and treatments of electrical stimulation have increased the thickness of adjacent muscles and reduced the subluxation. ${ }^{24}$ The excitability of permanently denervated muscles in humans decreases such that the muscles were considered unsuitable for the restoration of motor function by functional electrical stimulation. ${ }^{3}$ Electrical stimulation of denervated muscle can maintain the excitability of denervated muscles in rats. ${ }^{11}$ Treatments with electrical stimulation of permanently denervated muscles in the legs of humans have restored excitability to the muscles such that muscle hypertrophy, gain in strength, and ability to stand was demonstrated.20,21,27

In summary, the current study has determined the range in the number of tetanic contractions generated per day for denervated EDL muscles of rats for 5 weeks of stimulation. The same protocol is not expected to likewise maintain mass and force in other muscles that have different fiber compositions or architecture; nor would it be expected to be effective in other species of animals. Previous studies showed that a single protocol of stimulation applied to different muscles resulted in different levels of mass and force, ${ }^{12,16,18,33}$ and a single protocol applied to the same muscle in different species resulted in different levels of mass and force. ${ }^{23}$ Nevertheless, we have shown that appropriate protocols of electrically induced tetanic contractions maintain mass, 
force, and fiber size in at least one muscle type, the EDL muscle of rats.

This work was supported by NIH grant number PO1-AG10821, NIA training grant number AG00114, and the Contractility Core of Nathan Shock Center, grant number P30-AG13283. Dr. Cederna received support as the John E. Hoopes Academic Scholar of the American Association of Plastic Surgeons.

\section{REFERENCES}

1. Al-Amood WS, Lewis DM. Fast-to-slow transition in myosin heavy chain expression of rabbit muscle fibres induced by chronic low-frequency stimulation. J Physiol (Lond) 1987; 392:377-395

2. Al-Amood WS, Lewis DM, Schmalbuch H. Effects of chronic electrical stimulation on contractile properties of long-term denervated rat skeletal muscle. J Physiol (Lond) 1991;441: 243-256.

3. Betz RR, Mulcahey MJ, Smith BT, Triolo RJ, Weiss AA, Moynahan M, Keith MW, Peckham PH. Bipolar latissimus dorsi transposition and functional neuromuscular stimulation to restore elbow flexion in an individual with $\mathrm{C} 4$ quadriplegia and C5 denervation. J Spinal Cord Med 1992;15:220-228.

4. Buffelli M, Pasino E, Cangiano A. Paralysis of rat skeletal muscle equally affects contractile properties as does permanent denervation. J Muscle Res Cell Motil 1997;18:683-695.

5. Carlson BM, Billington L, Faulkner J. Studies on the regenerative recovery of long-term denervated muscle in rats. Restor Neurol Neurosci 1996;10:77-84.

6. Carlson BM, Faulkner JA. Muscle regeneration in young and old rats: effects of motor nerve transection with and without marcaine treatment. J Gerontol A Biol Sci Med Sci 1998;53A: B52-B57.

7. Carlson BM, Faulkner JA. Reinnervation of long-term denervated rat muscle freely grafted into an innervated limb. Exp Neurol 1988;102:50 -56.

8. Cole BG, Gardiner PF. Does electrical stimulation of denervated muscle, continued after reinnervation, influence recovery of contractile function? Exp Neurol 1984;85:52-62.

9. Dennis RG. Bipolar implantable stimulation for long-term denervated-muscle experiments. Med Biol Eng Comput 1998; $36: 225-228$

10. Dennis RG, Dow DE, Faulkner JA. An implantable device for stimulation of denervated muscles in rats. Med Eng Phys 2003;25:239-253.

11. Dennis RG, Dow DE, Hsueh A, Faulkner JA. Excitability of engineered muscle constructs, denervated and denervatedstimulated muscles of rats, and control skeletal muscles in neonatal, young, adult, and old mice and rats. Biophys J 2002;82:364A.

12. Eken T, Gundersen K. Electrical stimulation resembling normal motor-unit activity: effects on denervated fast and slow rat muscles. J Physiol (Lond) 1988;402:651-669.

13. Faulkner JA, Brooks SV, Dennis RG. Measurement of recovery of function following whole muscle transfer, myoblast transfer, and gene therapy. In: Morgan JR, Yarmush ML, editors. Tissue engineering methods and protocols. Totowa: Humana Press; 1999. p 155-172.

14. Finol HJ, Lewis DM, Owens R. The effects of denervation on contractile properties of rat skeletal muscle. J Physiol (Lond) 1981;319:81-92.

15. Fu SY, Gordon T. Contributing factors to poor functional recovery after delayed nerve repair: prolonged denervation. J Neurosci 1995;15:3886-3895.
16. Gundersen K, Eken T. The importance of frequency and amount of electrical stimulation for contractile properties of denervated rat muscles. Acta Physiol Scand 1992;145:49-57.

17. Gutmann E, Guttmann L. The effect of galvanic exercise on denervated and re-innervated muscle in the rabbit. J Neurol Neurosurg Psychiatry 1944;7:7-17.

18. Hennig R, Lomo T. Effects of chronic stimulation on the size and speed of long-term denervated and innervated rat fast and slow skeletal muscles. Acta Physiol Scand 1987;130:115131

19. Hennig R, Lomo T. Firing patterns of motor units in normal rats. Nature 1985;314:164-166.

20. Kern H, Hofer C, Modlin M, Forstner C, Mayr W, Richter W. Functional electrical stimulation (FES) of long-term denervated muscles in humans: clinical observations and laboratory findings. Basic Appl Myol 2002;12:291-299.

21. Kern H, Hofer C, Strohhofer M, Mayr W, Richter W, Stohr H Standing up with denervated muscles in humans using functional electrical stimulation. Artif Organs 1999;23:447-452.

22. Kobayashi J, Mackinnon SE, Watanabe O, Ball DJ, Gu XM, Hunter DA, Kuzon WM. The effect of duration of muscle denervation on functional recovery in the rat model. Muscle Nerve 1997;20:858-866.

23. Lewis DM, Al-Amood WS, Schmalbuch H. Effects of long-term phasic electrical stimulation on denervated soleus muscle: guinea-pig contrasted with rat. J Muscle Res Cell Motil 1997; 18:573-586.

24. Loeb GE, Peck RA, Moore WH, Hood K. BION system for distributed neural prosthetic interfaces. Med Eng Phys 2001; 23:9-18.

25. Lu DX, Huang SK, Carlson BM. Electron microscopic study of long-term denervated rat skeletal muscle. Anat Rec 1997;248: 355-365.

26. Mortimer JT, Kaufman D, Roessmann U. Intramuscular electrical stimulation: tissue damage. Ann Biomed Eng 1980;8: 235-244.

27. Neumayer C, Happak W, Kern H, Gruber H. Hypertrophy and transformation of muscle fibers in paraplegic patients. Artif Organs 1997;21:188-190.

28. Nicolaidis SC, Williams HB. Muscle preservation using an implantable electrical system after nerve injury and repair. Microsurgery 2001;21:241-247.

29. Nix WA. Effects of intermittent high frequency electrical stimulation on denervated EDL muscle of rabbit. Muscle Nerve 1990;13:580-585

30. Scheiner A, Mortimer JT, Roessmann U. Imbalanced biphasic electrical-stimulation-muscle-tissue damage. Ann Biomed Eng 1990;18:407-425.

31. Schmalbruch H, al Amood WS, Lewis DM. Morphology of long-term denervated rat soleus muscle and the effect of chronic electrical stimulation. J Physiol (Lond) 1991;441:233241.

32. Viguie CA, Lu DX, Huang SK, Rengen H, Carlson BM. Quantitative study of the effects of long-term denervation on the extensor digitorum longus muscle of the rat. Anat Rec 1997; 248:346-354.

33. Westgaard RH, Lomo T. Control of contractile properties within adaptive ranges by patterns of impulse activity in the rat. J Neurosci 1988;8:4415-4426.

34. Williams HB. The value of continuous electrical muscle stimulation using a completely implantable system in the preservation of muscle function following motor nerve injury and repair: an experimental study. Microsurgery 1996;17:589596. 